\title{
Optimizing Door Assignment in LTL-Terminals by Evolutionary Multiobjective Algorithms
}

\author{
Thomas Bartz-Beielstein, Annette Chmielewski, Michael Janas, \\ Boris Naujoks and Robert Scheffermann
}

\begin{abstract}
In less-than-truckload terminals arriving trucks have to be allocated to a gate and to a time slot for unloading. The allocation to a specific gate results in different transportation volumes for the forklift trucks inside of the terminal, depending on the destinations of the truck's loads. While minimizing these transports the time for trucks waiting to be ordered to a gate also has to be minimized. For the first time this problem has been tackled as a 2 -objective optimization problem and was solved by an (1+1)-evolution strategy. We developed a model which is derived from real freight forwarder's data and represents a small company's terminal on an average workday.
\end{abstract}

\section{INTRODUCTION}

In logistical terminals it is to be decided at which gate and at what time a truck should be unloaded. The goods have different destinations inside of the terminal and the distance from the gates to these destinations is different for each gate as illustrated in Fig. 1. It is important to minimize the waiting time for trucks and keep the transportation volume inside of the terminal low. Goods with a total weight under three tonnes, which are often placed on a pallet for further transport activities, are called less-than-truckload (LTL) consignments.

Bermudez \& Cole (2001) were one of the first tackling this kind of problem. They used a genetic algorithm to min In their model they assume that a single gate does serve only a single truck, which means just the allocation of trucks to gates is considered and no time constraints exist. Another approach by Stickel \& Furmans (2005) on crossdocking terminals concentrates on the time-scheduling aspect and also takes the vehicle routing for inbound and outbound routes into account. The associated mathematical model is very complex. It was possible to solve the mixed integer linear program (MILP) with CPlex for very small problem instances. The processes inside of terminals were solved by Li \& Rodrigues (2004) using an hybrid evolutionary algorithm.

Chmielewski \& Clausen $(2005,2006)$ developed an enhanced mathematical model for optimizing less-thantruckload terminals that is based on a time discrete multicommodity flow and supplemented by necessary side constraints. The resulting MILP was programmed with the optimization solver CPlex 4.1 and different test scenarios were applied to

Thomas Bartz-Beielstein, Michael Janas, and Boris Naujoks are with the Chair of Algorithm Engineering and Systems Analysis, University of Dortmund, 44221 Dortmund, Germany (email: \{thomas.bartz-beielstein, michael.janas, boris.naujoks\}@udo.edu)

Annette Chmielewski and Robert Scheffermann are with the Chair for Transportation Science, University of Dortmund, 44221 Dortmund, Germany (email: \{scheffermann, chmielewski\}@uni-dortmund.de) the Branch-and-Cut algorithm implemented in CPlex. On the one hand the test scenarios show that for small and middle sized problem instances good solutions can still be found. But on the other hand - especially for the case of medium sized problems-finding optimal solutions takes up to 30 minutes or more. Obviously, this time span is prohibitive for on-line optimization problems. So, exact solution methods are not relevant for the dynamic allocation of trucks to gates in logistical terminals. Also the two objectives have so far only been considered by including a penalty for late docking into the monocriterial objective function.

In our paper we present an approach that allows to allocate multiple trucks to the same gate on different timeslots, so extending the model Bermudez \& Cole (2001) used for their genetic algorithm. Compared to Stickel \& Furmans (2005) we were able to find good solutions for much larger problem instances, but the underlying model of crossdockingterminals is different in many aspects from LTL-terminals. Therefore, the results are not directly comparable. The underlying model is similar to the one used by Chmielewski \& Clausen (2005), but in our new approach the problem was tackled as a multiobjective problem for the first time.

We solved the two criteria decision problem of minimizing the transportation volume inside of LTL terminals and the waiting time for trucks between arrival at the terminal and being assigned to a gate. This problem will be referred to as the LTL-problem in the remainder of this article. The next section will give a more detailed definition of the corresponding model, which is very similar to the model used in Chmielewski \& Clausen (2005). Section 3 will introduce the algorithm we used to solve the problem: a $1+1$ evolution strategy. The experiments are then described in Sect. 4. Next the experiment's results are discussed in Sect. 5 and different variants are being compared. Finally we give a summary in Sect. 6 .

\section{Problem}

The transport of LTL goods within a country or a region is organized via a transportation network. The transportation request of one customer (normally between 1 and 10 pallets) usually does not suffice to fill the load area of a whole truck (up to 33 pallets). The network structure allows companies to use bundling effects by consolidating all consignments with the same long distance destination on one truck. Therefore, a transportation network consists of several logistical sites, which will be referred to as freight forwarding terminals. 
$\left[G_{i 1}, G_{i 2}, \ldots, G_{i m}\right]$, where $m$ denotes the number of gates. Each list $G_{i j}=\left[T_{i j 1}, \ldots, T_{i j k}\right]$ represents one gate. These list entries represent tours. Each tour $T_{i j l}$ consists of an array with four integer values:

$$
T_{i j l}=\text { [tourNumber, gateNumber, startTime, endTime]. }
$$

To accelerate the function evaluation, two additional arrays to store times and gates were implemented. Within the framework of evolutionary algorithms, candidate solutions are also called individuals.

\section{B. Description of the Simple Heuristic}

The existing heuristical approach mimics the decisions of a human planner and follows some simple rules and classification figures.

First the tours are sorted by a combination of priority and expected difficulty of being assigned to a gate. The tour's priority depends on an assesment based on the user's experience and mix/type of loads on the truck, while the difficulty depends on the size of the time window and time of arrival. The weight of each figure can be defined by the user to find a sorted list of tours matching the individual requirements.

Following this sorted lists each tour is then assigned to a gate at the earliest possible time. This is done by calculating the expected waiting time for the truck at each gate and the resulting transportation volume for the loads on the truck from that gate to all the load's destinations inside of the terminal. These two criterias are used to assign the tour to a gate and the user may define the weights for combining the two objectives to find a solution.

By changing a tour's priority or varying the heuristic's weights different solutions can be found focusing either on optimizing the waiting times for trucks and/or transportation volume inside of the terminal.

It has to be pointed out, that the heuristic is unable to optimize the allocation of outbound tours - they have to be assigned to gates manually beforehand. This is similar to the real world planning task when in LTL-terminals the allocation of outbound tours usually is historically grown and not adjusted regulary. Another drawback is, that the heuristic is not built to find a set of Pareto-optimal solutions. It has to be decided beforehand, if waiting times or transportation volume has to be considered or how these objectives should be weighted. So a single solution can be found but there is no further knowledge about the possible solution space available.

For these reasons, we decided to develop an algorithm that can tackle the problem in a better way. A multiobjective approach not only gives a range of solutions so that the human planner is able to pick one that satisfies his needswe also gather knowledge about the solutions possible to create.

A much more important issue is to develop an algorithm which is able to estimate the potential benefit when outbound tours can be allocated freely. As a result, the current allocation scheme and processes in LTL-terminals can be optimized.

\section{Description of the Evolution Strategy}

Evolutionary algorithms are well suited to satisfy our needs, as they can easily be implemented to find a set of Pareto-optimal solutions and work quite well even on very complex combinatorial optimization problems.

Therefore we have chosen a simple EA, the $(1+1)$-ES. Schwefel (1995) described this algorithm as "the minimal concept for an imitation of organic evolution." Let $f$ denote a multi-objective function to be minimized. The rules of an $(1+1)$-ES for MCO can be described as shown in Algorithm 1.

As already mentioned before, there are two objectives arising from the problem: minimization of distances within the minimization of waiting times. Due to this multi-objective nature of the problem, it was reasonable to apply multiobjective optimization techniques. The decision to invoke evolutionary multi-objective optimization techniques is based on the needs of decision makers (planners) to have a set of alternative solutions at hand to derive a final decision. Here, the concept of Pareto dominance comes into play.

A solution one is said to dominate a solution two, iff all components of the fitness function $f$ of solution one are not greater that the corresponding components of solution two and really smaller in at least one component. The set of non-dominated solutions is called the Pareto set of solutions while the corresponding pictures under function $f$ are called the Pareto front.

The appreciated set of alternative solutions, a Pareto set, to allow an a posterior decision in multi-objective optimisation problems is offered by evolutionary optimisation techniques (Deb, 2001; Coello Coello et al., 2002). Several other techniques need an a priori choice of a ranking of objectives or the definition of weights to start the optimization.

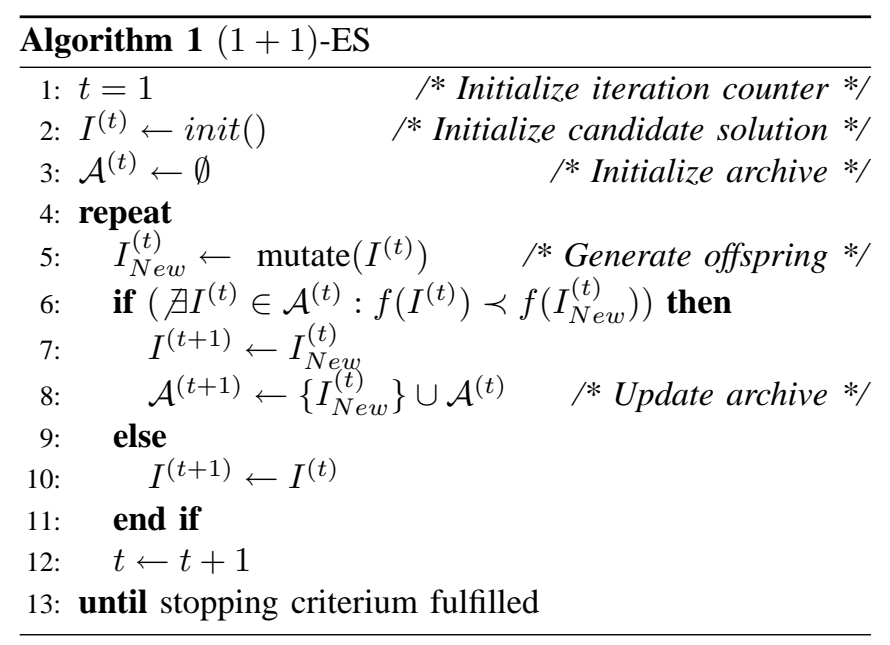

\section{Fitness Function}

As already mentioned while describing the problem, two objective functions $f_{1}, f_{2}$ are considered for minimization. 
Ignoring the resources needed for the operations inside the transfer stations, the first one describes the way of each pallet:

$$
f_{1}\left(I_{i}\right):=\sum_{j=1}^{m} \sum_{l=1}^{k} \sum_{r=1}^{s} d\left(G_{i j}\left(P_{i j l r}\right), G_{i d}\left(P_{i j l r}\right)\right),
$$

with $P_{i j l r}$ being r-th pallet of tour $T_{i j l}$ at gate $G_{i j}$ with destination gate $G_{i d}(d \in\{1, \ldots, m\})$. The function $d$ describes the distance inside the transfer station from one gate to another. It could also invoke different kinds of resources for the operations, but we limited ourselves to distances here.

The second objective function displays the waiting time for each truck:

$$
f_{2}\left(I_{i}\right):=\sum_{j=1}^{m} \sum_{l=1}^{k} t_{w}\left(T_{i j l}\right)
$$

with function $t_{w}\left(T_{i j l}\right)$ being the difference between the point of time the unloading of truck of the corresponding tour is started and the arrival time at the transfer station. This time is normally spent in some parking area. For reasons of simplicity, we neglected a detailed description of all constraints that can be derived from the problem description. Of course, all constraints are represented in our algorithm for the task.

\section{E. Problem Specific Operators for the 1+1-ES}

Search points are initialized as follows: Long distance tours $\left(\mathcal{T}_{\text {long }}\right)$ are randomly assigned to long distance gates $\left(\mathcal{G}_{\text {long }}\right)$. If all long distance gates are occupied, the remaining long distance tours are assigned to multifunctional gates. Short distance tours $\left(\mathcal{T}_{\text {short }}\right)$ are assigned to the first available gate from the set of short distance and multifunctional gates. The initialization is restarted if a tour cannot be assigned to any gate.

The mutation operator chooses randomly a tour $T \in$ $\left\{\mathcal{T}_{\text {long }} \cup \mathcal{T}_{\text {short }}\right\}$, which will be reassigned. Next, a gate $G^{\prime} \in \mathcal{G}$ with feasible arrival time is selected randomly. Two mutation opererators have been implemented:

1) Random Mutation: redistribute tours $T^{\prime}$ that have been previously assigned to $G^{\prime}$ randomly to available gates.

2) Quick Mutation: assign $T^{\prime}$ to the first available gate.

In both cases, the mutation is repeated if a tour cannot be assigned.

\section{F. Selection}

As mentioned above, we utilized a simple $(1+1)$-ES selection scheme, but it has to deal with multiple objectives and therefore differs from the single-objective case, of course. The selection scheme implemented accepts the offspring individual to become the parent in the next generation, iff it is non-dominated by all individuals generated by the algorithms until now. The set of individuals generated within the optimization run and non-dominated by each other is called the current Pareto front $\mathrm{PF}_{\text {cur }}$.
In contrast to the simple evolutionary multi-objective optimizer SEMO, our approach keeps the parent individual, if the offspring individual is not selected. SEMO chooses a new parent in each generation uniformly from $\mathrm{PF}_{\text {cur }}$ (Laumanns, 2003). The current Pareto front is updated after each generation, individuals dominated by the new parent are removed.

\section{EXPERIMENTS}

An experimental design has to be specified before the experimental analysis can be started. Our experiments are based on the experimental methodology from Bartz-Beielstein (2006). A hypervolume can be used to judge the performance of algorithms for multiobjective optimization problems. To calculate the hypervolume value $\mathcal{S}\left(\mathrm{PF}_{\text {cur }}\right)$ the objective function values of each individual of the Pareto-front are considered. The hypervolume is the space covered by the solutions of the Pareto front calculated with respect to a chosen reference point $x^{r e f}$ :

$$
\mathcal{S}(\mathrm{PF})=\Delta\left(\bigcup_{I}\left\{x \in \mathbb{R}^{n} \mid f(I) \prec x \prec x^{r e f}\right\}\right),
$$

with $\Delta$ being the Lebesque measure of the hypercube spanned by the solutions from the Pareto-front and the reference point. For the two-dimensional case studied here, this can be simplified to:

$$
\mathcal{S}(\mathrm{PF})=\Delta\left(\bigcup_{I}\left[x_{1}^{r e f}-f_{1}(I)\right] \times\left[x_{2}^{r e f}-f_{2}(I)\right]\right) .
$$

As the door-assignment problem was introduced as a new problem class, no representative results (as for TSP instances) are available. To overcome this difficulty, we proceeded as follows: The problem was solved with several algorithms that used a similar budget, i.e., number of function evaluations. The upper $10 \%$ quantile of the function values from all results was chosen to characterize "good" algorithms. Runlength distributions (Fig. 2) as proposed by Hoos (1998) were used to determine an adequate number of function evaluations for the final comparisons.

They are reliable tools to avoid floor- and ceiling effects. These effects occur if problem instances that are chosen, which are too hard, or too easy, respectively, for the algorithms under consideration.

As can be seen from Fig. 2, 300,000 function evaluations are a good compromise to detect differences between algorithms and to enable a fair comparison.

One main research topic in evolutionary computation is the design of problem-specific evolutionary algorithms (Beielstein et al., 2003). The aim is to systematize the design of evolutionary algorithms for problems with nonstandard representations. Especially nonstandard, problem-specific representations and variation operators are of great importance. Therefore, it is an important step to develop and analyze mutation operators for the LTL-problem. Two mutation (variation) operators, which were introduced in Sect. III-E, are subject of our experimental analysis. Our comparison is 
further investigated and compared to other techniques. The $(1+1)$-ES shares some properties of the SEMO algorithm. It suggests itself, that these approaches are to be compared on the current test problem as well as on other ones.

Moreover, the $(1+1)$-ES will be compared to the multimembered $(\mu+\lambda)$-ES. For the selection in the current multiobjective test case, individuals will be ranked according to their dominance-rank in comparison to all other $\mu+\lambda-1$ individuals. If it has to be decided between different solutions with the same dominance-rank, this is done uniformly distributed at random. And, we did not apply the very efficient and effective sequential parameter optimization technique to improve the performance of the $(\mu+\lambda)$-ES, see BartzBeielstein (2006). This tuning procedure will be integrated into further analyses.

Furthermore, new approaches to solve smaller instances of the current problem mathematically are under development and will be investigated and compared to the approach at hand in the near future. A detailed comparison will be done to deduce in what strategy should be preferred in which cases. This is the most important conclusion for operators. But it leads to more restrictions concerning gates if handled within the mathematical approach. For a reliable comparison, this needs to be treated in the evolutionary algorithm as well. On the other hand side, the evolutionary approach offering more flexible solutions without the need to fix gates to special tours is highly appreciated by the operators.

\section{ACKNOWLEDGMENT}

This work was supported by the Deutsche Forschungsgemeinschaft (DFG) as part of the Collaborative Research Center "Computational Intelligence (531)."

\section{REFERENCES}

Bartz-Beielstein, T. (2006). Experimental Research in Evolutionary Computation-The New Experimentalism. Berlin, Heidelberg, New York: Springer.

Beielstein, T., Mehnen, J., Schönemann, L., Schwefel, H.P., Surmann, T., Weinert, K., \& Wiesmann, D. (2003). Design of evolutionary algorithms and applications in surface reconstruction. In H.-P. Schwefel, I. Wegener, \& K. Weinert (Eds.), Advances in Computational IntelligenceTheory and Practice (pp. 145-193). Berlin, Heidelberg, New York: Springer.

Bermudez, R. \& Cole, M. H. (2001). A genetic algorithm approach to door assignments in breakbulk terminals. Technical Report MBTC-1102, Mack-Blackwell Transportation Center, University of Arkansas, Fayetteville, Arkansas.

Chmielewski, A. \& Clausen, U. (2005). Entwicklung eines Dispositionsleitstandes zur Bestimmung optimaler Torbelegungen in Stückgutspeditionsanlagen. WGTL Logictics Journal.

Chmielewski, A. \& Clausen, U. (2006). Entwicklung optimaler Torbelegungspläne in Stückgutspeditionsanlagen. In DSOR Contributions to Information Systems, MKWI 2006, Information Systems in Transport and Traffic.

Coello Coello, C. A., Van Veldhuizen, D. A., \& Lamont, G. B. (2002). Evolutionary Algorithms for Solving MultiObjective Problems. Kluwer, New York.

Deb, K. (2001). Multi-Objective Optimization using Evolutionary Algorithms. Wiley-Interscience Series in Systems and Optimization. New York NY: Wiley.

Hoos, H. H. (1998). Stochastic Local Search-Methods, Models, Applications. PhD thesis, Technische Universität Darmstadt, Germany.

Laumanns, M. (2003). Analysis and Applications of Evolutionary Multiobjective Optimization Algorithms. $\mathrm{PhD}$ thesis, Swiss Federal Institute of Technology, Zürich, Switzerland.

Li, Y. \& Rodrigues, B. (2004). Crossdocking - jit scheduling with time windows. Journal of Operational Research Society, 10, 1-10.

Schwefel, H.-P. (1995). Evolution and Optimum Seeking. Sixth-Generation Computer Technology. New York NY: Wiley.

Stickel, M. \& Furmans, K. (2005). An optimal control policy for crossdocking terminals. In Proc. of the International Conference of Operations Research 2005: Gesellschaft für Operations Research (GOR) Springer, Berlin. (accepted for publication). 